\title{
COMBINATORIAL EQUIVALENCE BETWEEN GROUP PRESENTATIONS
}

\author{
SUSHIL JAJODIA
}

ABstract. Let

$$
P=\left(x_{1}, \ldots, x_{n}: W_{1}, \ldots, W_{m}\right) \text { and } R=\left(x_{1}, \ldots, x_{n}: R_{1}, \ldots, R_{m}\right)
$$

be two presentations, with the same generators, for a group $\pi$. In this note, we give a necessary and sufficient criterion which insures the existence of a combinatorial equivalence between $P$ and $R$ requiring only replacement operations.

I. Introduction. Let

$$
P=\left(x_{1}, \ldots, x_{n}: W_{1}, \ldots, W_{m}\right)
$$

and

$$
R=\left(x_{1}, \ldots, x_{n}: R_{1}, \ldots, R_{m}\right)
$$

be two presentations, with the same generators, for a group $\pi$ (i.e., the normal closures of $W_{1}, \ldots, W_{m}$ and of $R_{1}, \ldots, R_{m}$ in the free group $F$ on $x_{1}, \ldots, x_{n}$ coincide). In [8, p. 95], A. J. Sieradski makes the following conjecture: Suppose there exists a combinatorial equivalence between $P$ and $R$ inducing the identity isomorphism. Then there exists a combinatorial equivalence between $P$ and $R$ requiring only replacement operations. We are unable to prove this conjecture; however, we give a necessary and sufficient criterion which insures its existence.

II. Definition of combinatorial equivalence. Let $\pi$ be any group, and let

$$
R=\left(x_{1}, \ldots, x_{n}: R_{1}, \ldots, R_{m}\right)=\left(x_{i}: R_{j}\right)
$$

be a finite presentation for $\pi$. We have the following combinatorial operations on the presentation $R$ :

(1) The expansion operation. It appends to the presentation $R$ a new generator $x \notin\left\{x_{i}\right\}$ and a relator $W x^{-1}$ where $W$ is an element in the free group $F$ on generators $x_{1}, \ldots, x_{n}$. Therefore

$$
\left(x_{i}: R_{j}\right) \rightarrow\left(x_{i}, x: R_{j}, W x^{-1}\right) .
$$

(2) The contraction operation. It simultaneously deletes a generator $x$ and a relator $W x^{-1}$ provided that $W$ and the remaining relators $R_{j}$ are elements in the free group $F$ generated by the remaining generators $x_{1}, \ldots, x_{n}$. Therefore $\left(x_{i}, x: R_{j}, W x^{-1}\right) \rightarrow\left(x_{i}: R_{j}\right)$.

(3) The replacement operation. It replaces a single relator $R$ by a new relator $S$ provided that $S$ and $R^{ \pm 1}$ are conjugates modulo the normal subgroup $N\left(R_{j}\right)$ of $F$

Received by the editors June 10, 1981.

1980 Mathematics Subject Classification. Primary $20 \mathrm{E} 06$. 
generated by the other relators $R_{j}$ which are kept unchanged. Hence $\left(x_{i}: R_{j}, R\right) \rightarrow$ $\left(x_{i}: R_{j}, S\right)$.

Let $P$ and $R$ be two presentations for the same group $\pi$. We say $P$ and $R$ are combinatorially equivalent if there is a sequence

$$
P=P_{0} \rightarrow P_{1} \rightarrow \cdots \rightarrow P_{s-1} \rightarrow P_{s}=R
$$

of combinatorial operations which begin with $P$ and end with $R$.

Note that we do not permit the Tietze operation of adding trivial relators to the presentation. This restriction is motivated by topological considerations. We urge the reader to read [8] for details.

III. Necessary and sufficient criterion. Suppose the presentations (1) and (2), with the same deficiency and the same generators, present the same group $\pi$. We will now formulate the necessary and sufficient conditions.

Let $F$ denote the free group generated by $x_{1}, \ldots, x_{n}$ and $R$ the normal closure in $F$ of $R_{1}, \ldots, R_{m}$. Let $\bar{F}$ denote the free group on symbols $r_{1}, \ldots, r_{m}$. Since each $W_{i} \in R$, we can write

$$
W_{i}=\prod_{k=1}^{m_{i}}\left(x_{i k} R_{i k} x_{i k}^{-1}\right)^{\epsilon_{i k}}
$$

where $R_{i k}=R_{j}$ for some $j, 1 \leq j \leq m, x_{i k} \in F$, and $\epsilon_{i k}= \pm 1$. Let

$$
w_{i}=\prod_{k=1}^{m_{i}}\left(x_{i k} r_{i k} x_{i k}^{-1}\right)^{\epsilon_{i k}}
$$

where if $R_{i k}=R_{j}$ in $W_{i}$, then $r_{i k}=r_{j}$ in $w_{i}$.

Let $J$ denote the $m \times m$ matrix

$$
J=\left\|\begin{array}{ccc}
\frac{\partial w_{1}}{\partial r_{1}} & \cdots & \frac{\partial w_{1}}{\partial r_{m}} \\
\vdots & & \vdots \\
\frac{\partial w_{m}}{\partial r_{1}} & \cdots & \frac{\partial w_{m}}{\partial r_{m}}
\end{array}\right\|
$$

where $\partial / \partial r_{j}: Z F \rightarrow Z F$ denotes the $j$ th free partial derivative [2].

THEOREM. Notation as above. Then the following are equivalent.

(1) There is a combinatorial equivalence between $P$ and $R$ requiring only replacement operations.

(2) $\left\{x_{1}, \ldots, x_{n}, w_{1}, \ldots, w_{m}\right\}$ forms a generating set for the free product $F * \bar{F}$.

(3) $J$ has a right inverse.

ProOF. (1) $\Rightarrow$ (2) Suppose $P$ is combinatorially equivalent to $R$ using only replacement operations. Now all replacement operations are composites of these three replacement operations $[8$, p. 69]:

(a) Replace a single relator $R_{i}$ by its inverse $R_{i}^{-1}$.

(b) Replace a relator $R_{i}$ by the product $R_{i} R_{j}$ or $R_{j} R_{i}$ with a different relator $R_{j}$.

(c) Replace a relator $R_{i}$ by $x^{\epsilon} R_{i} x^{-\epsilon}$ by a generator $x, \epsilon= \pm 1$. 
This means that we can convert $x_{1}, \ldots, x_{n}, r_{1}, \ldots, r_{m}$ into $x_{1}, \ldots, x_{n}, w_{1}, \ldots, w_{m}$ using transformations which leave $x_{1}, \ldots, x_{n}$ fixed and on the remaining generators the following operations:

(a') Replace $r$ by $r^{\prime}$.

(b') Replace $r$ by $r \bar{r}$ or $\bar{r} r$ where $\bar{r}$ is a different generator.

(c) Replace $r$ by $x^{\epsilon} r x^{-\epsilon}$ where $x=x_{i}, 1 \leq i \leq n$.

But these are the elementary Nielsen transformations [3, p. 130]. Hence $x_{1}, \ldots, x_{n}$, $w_{1}, \ldots, w_{m}$ forms a generating set for $F * \bar{F}$.

(2) $\Rightarrow(1)$ Suppose $\left\{x_{1}, \ldots, x_{n}, w_{1}, \ldots, w_{m}\right\}$ generates $F * \bar{F}$. Then by a result of Rapaport [7, §5], it follows that one can obtain $x_{1}, \ldots, x_{n}, w_{1}, \ldots, w_{m}$ from $x_{1}, \ldots, x_{n}, r_{1}, \ldots, r_{m}$ using transformations which leave $x_{1}, \ldots, x_{n}$ fixed and on the remaining generators the operations $\left(a^{\prime}\right),\left(b^{\prime}\right)$, and $\left(c^{\prime}\right)$ described above. But then one can construct a combinatorial equivalence between $P$ and $R$ using only replacement operations.

$(2) \Rightarrow(3)$ Suppose $\left\{x_{1}, \ldots, x_{n}, w_{1}, \ldots, w_{m}\right\}$ forms a generating set for $F * \bar{F}$. Then by the Inverse Function Theorem [1, p. 635], the Jacobian which has the form

$$
\left\|\begin{array}{ll}
I_{n} & 0 \\
A & J
\end{array}\right\|
$$

has a right inverse $B$. By a result of Kaplansky [6], $B$ is a two-sided inverse. Therefore $J$ has a right inverse.

$(3) \Rightarrow(2)$ Suppose $J$ has a right inverse $H$. Then the Jacobian which has the form

$$
\left\|\begin{array}{ll}
I_{n} & 0_{m} \\
A_{m \times n} & J_{m}
\end{array}\right\|
$$

has a right inverse

$$
\left\|\begin{array}{cc}
I_{n} & 0_{m} \\
-H_{m} A_{m \times n} & H_{m}
\end{array}\right\| \text {. }
$$

The result now follows from the Inverse Function Theorem [1, p. 635]. This completes the proof.

EXAMPLE. Consider the two presentations

$$
P=\left(x, y, z: W_{1}=x^{5}, W_{2}=y^{5}, W_{3}=z^{5}, W_{r}=[x, y], W_{5}=[x, z], W_{6}=[y, z]\right)
$$

and

$$
R=\left(x, y, z: R_{1}=x^{5}, R_{2}=y^{5}, R_{3}=z^{5}, R_{4}=\left[x^{4}, y\right], R_{5}=[x, z], R_{6}=[y, z]\right)
$$

of the group $Z_{5} \times Z_{5} \times Z_{5}$. We can write

$$
W_{4}=x y R_{1}^{-1} y^{-1} R_{4}^{-1} R_{1} x^{-1} \text {. }
$$

Therefore, $w_{4}=x y r^{-1} y^{-1} r_{4}^{-1} r_{1} x^{-1}$.

Clearly $\left\{x, y, z, w_{1}, w_{2}, w_{3}, w_{4}, w_{5}\right\}$ forms a generating set for $F * \bar{F}$ and the matrix $J$ is invertible. Thus there is a combinatorial equivalence between $P$ and $R$ using only replacement operations.

REMARK. In [4 and 5], examples are given of presentations which are combinatorially equivalent, but there does not exist a combinatorial equivalence between them requiring replacement operations only; however, these presentations do not have the same generators. 


\section{REFERENCES}

1. J. S. Birman, An inverse function theorem for free groups, Proc. Amer. Math. Soc. 41 (1973), 634-638.

2. R. H. Fox, Free differential calculus. I. Derivation in the free group ring, Ann. of Math. (2) 57 (1953), 547-560.

3. W. Magnus, A. Karrass and D. Solitar, Combinatorial group theory, Interscience, New York, 1966.

4. J. McCool and A. Pietrowski, On free products with amalgamation of two infinite cyclic groups, J. Algebra 18 (1971), 377-383.

5. W. Metzler, Über den homotopietyp zweidimensionaler $C W$-Komplexes und Elementartransformationen bei definierende relatumen, J. Reine Angew. Math. 285 (1976), 7-23.

6. M. S. Montgomery, Left and right inverses in group algebras, Bull. Amer. Math. Soc. 75 (1969), 539-540.

7. E. S. Rapaport, Groups of order 1: Some properties of presentations, Acta Math. 121 (1968), 127-150.

8. A. J. Sieradski, Combinatorial isomorphisms and combinatorial homotopy equivalences, J. Pure Appl. Algebra 7 (1976), 59-95.

COMPUTER SCIENCE Department, 304 MAthematical Sciences BuIlding, UNIVERSITY of MisSOURI-COLUMBIA, COLUMBIA, MisSOURI 65211 